T. OTSUKI

KODAI MATH. J.

5 (1982), 454-481

\title{
A CERTAIN PROPERTY OF GEODESICS OF THE FAMILY OF RIEMANNIAN MANIFOLDS $O_{n}^{2}(V)$
}

\author{
By TOMINOSUKE OTSUKI
}

\section{Introduction.}

This is exactly a continuation of Part (IV) ([14]) with the same title written by the present author. We shall use the same notation in it.

The period $T$ of any non-constant solution $x(t)$ of the non-linear differential equation of order 2 :

$$
n x\left(1-x^{2}\right) \frac{d^{2} x}{d t^{2}}+\left(\frac{d x}{d t}\right)^{2}+\left(1-x^{2}\right)\left(n x^{2}-1\right)=0
$$

with a constant $n>1$ such that $x^{2}+x^{\prime 2}<1$ is given by the integral:

$$
T=\sqrt{n c} \int_{x_{0}}^{x_{1}} \frac{d x}{x \sqrt{(n-x)\left\{x(n-x)^{n-1}-c\right\}}},
$$

where $0<x_{0}<1<x_{1}<n$ and $c=x_{0}\left(n-x_{0}\right)^{n-1}=x_{1}\left(n-x_{1}\right)^{n-1}$.

We proved in the last 10 th section of Part (IV) that the following conjecture is true for $n \geqq 84$ and shall show that it is also true for $16 \leqq n<84$ in the present paper.

Conjecture C. The period function $T$ as a function of $\tau=\left(x_{1}-1\right) /(n-1)$ and $n$ is monotone decreasing with respect to $n(>2)$ for any fixed $\tau(0<\tau<1)$.

The section numbers of this paper will start from 11 for convenience sake and so the section numbers from 1 to 10 mean the ones in Part (IV).

\section{$\S 11$. The fundamental principle to attain the purpose.}

Setting $T=\Omega(\tau, n)$, we have the two formulas

$$
\frac{\partial \Omega(\tau, n)}{\partial n}=-\frac{\sqrt{c}}{2 b^{2} \sqrt{n}} \int_{x_{0}}^{1} \frac{(1-x) W\left(x, x_{1}\right) d x}{x(n-x) \sqrt{x(n-x)^{n-1}-c}}
$$

where $b=\sqrt{ } B-c, B=(n-1)^{n-1}$ and

Received November 2, 1981 


$$
\begin{aligned}
W\left(x, x_{1}\right):= & \frac{x \sqrt{n-x} f_{0}(x)}{(x-1)^{3}}\{\lambda(X)-\lambda(x)\} . \\
& +\left[\frac{X \sqrt{n-X} f_{0}(X)}{(X-1)^{3}}-\frac{x \sqrt{n-x} f_{0}(x)}{(x-1)^{3}}\right]\left\{\frac{n}{n-1} \frac{\left(x_{1}-1\right)^{2}}{x_{1}\left(n-x_{1}\right)}-\lambda\left(x_{1}\right)+\lambda(X)\right\} \\
& -\left[\frac{X^{2} f_{1}(X)}{(1-X)^{3} \sqrt{n-X}}-\frac{x^{2} f_{1}(x)}{(1-x)^{3} \sqrt{n-x}}\right] \frac{\phi(x)-\psi\left(x_{1}\right)}{n \psi(x)},
\end{aligned}
$$

where $X=X(x), \phi(x)=x(n-x)^{n-1}$ (Lemma 3.1 in (III)) and

$$
\frac{\partial \Omega(\tau, n)}{\partial n}=-\frac{\sqrt{c / n}}{2 b^{2} n} \int_{x_{0}}^{1} \frac{(1-x) \sqrt{x(n-x)^{n-1}-c}}{x^{2}(n-x)^{n}} V\left(x, x_{1}\right) d x
$$

((7.4) and Proposition 3 in (III) and (1.1)).

In order to prove $\frac{\partial \Omega(\tau, n)}{\partial n}<0$, we have tried to prove $V\left(x, x_{1}\right)>0$ for $X(x)$ $<x_{1}<n$ and succeeded in it in the case $n \geqq 84$.

Using the notation

$$
\alpha_{0}=\alpha_{0}(n):=X_{n}^{-1}(2), \quad \alpha_{1}=\alpha_{1}(n):=X_{n}^{-1}\left(\frac{2 n+10}{2 n+1}\right),
$$

we have the following.

LEMMA 11.1. When $n \geqq 6$, we have

$$
V\left(x, x_{1}\right)>0 \text { for } 0<x \leqq \alpha_{0} \text { and } X(x) \leqq x_{1}<n .
$$

Proof. Since $V(x ; x)$ is increasing with respect to $x_{1}$ by Lemma 8.1 in (III) for $0<x<1$ and

$$
V(x, X(x))=U_{0}(x)-U_{1}(x)+U_{2}(x)+U_{4}(x)+U_{5}(x)-U_{6}(x),
$$

where $U_{i}(x), i=0,1,2,4,5,6$, are all positive for $0<x<1$, by Proposition 1 in (II), Lemma 2.1 in (II) and Lemma 7.1 in (III), it is sufficient to prove that

$$
V(x, X(x))>0 \quad \text { for } 0<x \leqq \alpha_{0} .
$$

When $n>2$, we have by means of Lemma 2.5

$$
\begin{aligned}
& -U_{1}(x)+U_{2}(x)+U_{4}(x)+U_{5}(x)-U_{6}(x) \\
& \quad>-\frac{n X}{(n-1)(X-1)^{3} \sqrt{ } n-X}=\left\{G_{2}(X)+3(X-1) f_{0}(X)\right\} \quad \text { for } \quad 0<x<1,
\end{aligned}
$$

and when $n \geqq 6$, we have by means of Lemma 3.1

$$
G_{2}(x)+3(x-1) f_{0}(x)>0 \quad \text { for } 2 \leqq x<n .
$$

Thus, we obtain the inequality (11.3).

Q.E. D.

LEMMA 11.2. When $n>13$, we have 


$$
V\left(x, x_{1}\right)>0 \quad \text { for } \quad \alpha_{0}<x \leqq \alpha_{1} \text { and } X(x) \leqq x_{1}<n .
$$

Proof. We obtain this from (11.2), (11.4) and Proposition 3 in (IV).

Q.E.D.

LEMMA 11.3. When $n \geqq 12$, we have $\frac{2 n+10}{2 n+1}<\beta$, where $\beta$ is the root of the quadratic equation: $(n-1) x^{2}+2 x-2 n=0$, which is given by

$$
\beta:=\frac{-1+\sqrt{2 n^{2}-2 n+1}}{n-1} .
$$

Proof. Since we have

$$
\begin{aligned}
{\left[(n-1) x^{2}+2 x-2 n\right]_{x=\frac{2 n+10}{2 n+1}} } & =\frac{4(n-1)(n+5)^{2}}{(2 n+1)^{2}}+\frac{4(n+5)}{2 n+1}-2 n \\
& =-\frac{2}{(2 n+1)^{2}} \cdot\left(2 n^{3}-18 n^{2}-51 n+40\right),
\end{aligned}
$$

it is sufficient to prove

$$
2 n^{3}-18 n^{2}-51 n+40>0 \text { for } n \geqq 12 .
$$

Now, setting $n=m+11$, we have easily

$$
\begin{aligned}
& 2 n^{3}-18 n^{2}-51 n+40 \\
& =2 m^{3}+48 m^{2}+279 m-37>0 \quad \text { for } m \geqq 1 . \quad \text { Q.E. D. }
\end{aligned}
$$

Remark. We know the fact on $\beta$ as follows: When $n>1$, we have

$$
\sqrt{\frac{2 n}{n+1}}<\beta<\sqrt{2}
$$

by Lemma 3.6 in (III). Since we have

and

$$
\left[2 m^{3}+48 m^{2}+279 m-37\right]_{m=0.13}=0.085594>0
$$

$$
\left[2 m^{3}+48 m^{2}+279 m-37\right]_{m=0.12}=-2.825344<0,
$$

we can insist the inequality in the above lemma for $n \geqq 11.13$ and not do it when $n=11.12$.

LEMMA 11.4. When $n \geqq 11$, we have

$$
\frac{\partial \Omega(\tau, n)}{\partial n}<0 \quad \text { for } \quad 1<x_{1} \leqq \beta .
$$

Proof. By means of Proposition 1 in (III) we have

$$
W\left(x, x_{1}\right)>0 \text { for } X^{-1}(\beta) \leqq x<1 \text { and } X(x) \leqq x_{1}<n
$$

when $n \geqq 11$. Hence, we obtain this lemma from (11.1).

Q.E.D. 


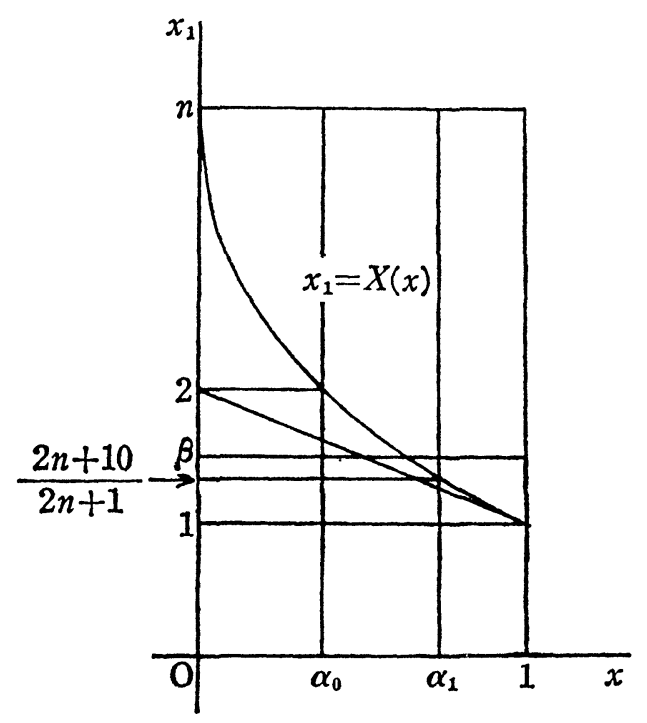

By virtue of Lemma $11.1 \sim$ Lemma 11.4 and the property of $V\left(x, x_{1}\right)$ that it is increasing with respect to $x_{1}$, we can obtain the inequality:

$$
\frac{\partial \Omega(\tau, n)}{\partial n}<0 \quad \text { for } \beta \leqq x_{1}<n,
$$

when $n \geqq 13$, if we can prove the inequality:

$$
V(x, \beta) \geqq 0 \quad \text { for } \quad \alpha_{1} \leqq x<1 .
$$

Finally, we express the function $V(x, \beta)$ in a convenient form for our purpose. From (1.2) (1.8) we have

$$
\begin{aligned}
V\left(x, x_{1}\right) & =\frac{x^{2} N\left(x, x_{1}\right)}{(1-x)^{5} \sqrt{n-x}}+\frac{X^{2} N\left(X, x_{1}\right)}{(X-1)^{5} \sqrt{n-X}} \\
& =\frac{x^{2} \sqrt{n-x} F_{2}(x)\left\{\lambda(x)-\tilde{\lambda}\left(x_{1}\right)\right\}}{(1-x)^{5}}-\frac{3 x^{2} f_{0}(x)}{(x-1)^{3} \sqrt{n-x}}+\frac{2 n x^{2}\{B-\psi(x)\}}{(1-x)^{2} \sqrt{n-x}} \\
& +\frac{X^{2} \sqrt{n-X} F_{2}(X)\left\{\lambda(X)-\tilde{\lambda}\left(x_{1}\right)\right\}}{(X-1)^{5}}+\frac{3 X^{2} f_{0}(X)}{(X-1)^{3} \sqrt{n-X}}-\frac{2 n X^{2}\{B-\phi(X)\}}{(X-1)^{2} \sqrt{n-X}}
\end{aligned}
$$

and by the definitions of $U_{i}(x),(1.9) \sim(1.13)$, we obtain

$$
\begin{aligned}
& =U_{0}(x)-U_{1}(x)+U_{2}(x)+\frac{x^{2} \sqrt{n-x} F_{2}(x)}{(1-x)^{5}}\left\{\tilde{\lambda}(X)-\tilde{\lambda}\left(x_{1}\right)\right\} \\
& \quad+U_{4}(x)+U_{5}(x)-U_{6}(x)+\frac{X^{2} \sqrt{n-X} F_{2}(X)}{(X-1)^{5}}\left\{\tilde{\lambda}(X)-\tilde{\lambda}\left(x_{1}\right)\right\},
\end{aligned}
$$

hence 


$$
\begin{aligned}
& V(x, \beta)=V(x, X(x)) \\
& \quad+\left\{\frac{x^{2} \sqrt{n-x} F_{2}(x)}{(1-x)^{5}}+\frac{X^{2} \sqrt{n-X F_{2}}(X)}{(X-1)^{5}}\right\}\{\tilde{\lambda}(X)-\tilde{\lambda}(\beta)\} .
\end{aligned}
$$

$\S 12$. Some evaluations related with $\tilde{\lambda}(x)$ and $F_{2}(x)$.

Regarding $V(x, X(x))$, we have the following evaluation: when $n \geqq 10$,

$$
\begin{gathered}
V(x, X(x))>\frac{s B \sqrt{n-1}}{100}(9.0651 n-379.1388 \times 2) \\
\text { for } \alpha_{1}(n) \leqq x<1,
\end{gathered}
$$

where $s=X(x)-1$.

LEMMA 12.1. When $n \geqq 12$, we have

$$
\begin{aligned}
& \frac{\tilde{\lambda}(X)-\tilde{\lambda}(\beta)}{X-1} \\
& >\frac{\left(2 n^{4}+2 n^{3}-38 n^{2}+83 n-40\right) \beta-n\left(2 n^{3}+4 n^{2}+43 n-40\right)}{18(2 n+1)\left\{n\left(n^{2}-n+2\right)-2\left(n^{2}-n+1\right) \beta\right\}} \\
& \text { for } 1<X \leqq \frac{2 n+10}{2 n+1} .
\end{aligned}
$$

Proof. Since we have

$$
\tilde{\lambda}^{\prime}(x)=-\frac{(x-1)\{n+(n-1) x\}}{(n-1) x^{2}(n-x)}
$$

by Lemma 7.1 in (III), we obtain

$$
\tilde{\lambda}(X)-\tilde{\lambda}(\beta)=\int_{\beta}^{X} \tilde{\lambda}^{\prime}(t) d t=\frac{1}{n-1} \int_{X}^{\beta} \frac{(t-1)\{n+(n-1) t\} d t}{t^{2}(n-t)} .
$$

Now, we show that the function $\frac{n+(n-1) t}{t^{2}(n-t)}$ is decreasing with respect to $t$ for $1 \leqq t \leqq 2$. In fact, we have

$$
\frac{d}{d t} \frac{n+(n-1) t}{t^{2}(n-t)}=\frac{-1}{t^{3}(n-t)^{2}}\left\{2 n^{2}+n(n-4) t-2(n-1) t^{2}\right\}
$$

and

hence

$$
2 n^{2}+n(n-4) t-2(n-1) t^{2}>0 \quad \text { for } \quad 0<t \leqq 2
$$

$$
\frac{d}{d t} \frac{n+(n-1) t}{t^{2}(n-t)}<0 \quad \text { for } \quad 1 \leqq t \leqq 2
$$

Thus we obtain

$$
\tilde{\lambda}(X)-\tilde{\lambda}(\beta)>\frac{n+(n-1) \beta}{(n-1) \beta^{2}(n-\beta)} \int_{X}^{\beta}(t-1) d t .
$$


Next, by Lemma 11.3 we obtain for $1<X \leqq \frac{2 n+10}{2 n+1}$

$$
\begin{aligned}
& \frac{1}{X-1} \int_{X}^{\beta}(t-1) d t=\frac{1}{2}\left\{\frac{(\beta-1)^{2}}{X-1}-(X-1)\right\} \\
& >\frac{1}{2}\left\{\frac{(\beta-1)^{2}}{t-1}-(t-1)\right\}_{t=-\frac{2 n+10}{2 n+1}} \\
& =\frac{(2 n+1)^{2}(\beta-1)^{2}-81}{18(2 n+1)}
\end{aligned}
$$

and

$$
\begin{aligned}
(2 n+1)^{2}(\beta-1)^{2}-81 & =(2 n+1)^{2} \cdot \frac{2(n-\beta)}{n-1}-2(2 n+1)^{2} \beta+(2 n+1)^{2}-81 \\
& =\frac{2}{n-1} \cdot\left\{6 n^{3}+4 n^{2}-41 n+40-n(2 n+1)^{2} \beta\right\},
\end{aligned}
$$

from which we get the inequality

$$
\frac{1}{X-1} \int_{X}^{\beta}(t-1) d t>\frac{6 n^{3}+4 n^{2}-41 n+40-n(2 n+1)^{2} \beta}{9(n-1)(2 n+1)} .
$$

Therefore we have

$$
\frac{\tilde{\lambda}(X)-\tilde{\lambda}(\beta)}{X-1}>\frac{n+(n-1) \beta}{2(n-\beta)^{2}} \cdot \frac{6 n^{3}+4 n^{2}-41 n+40-n(2 n+1)^{2} \beta}{9(n-1)(2 n+1)}
$$

On the other hand, using $(n-1) \beta^{2}=2(n-\beta)$, we obtain

and

$$
\begin{aligned}
(n-1)(n-\beta)^{2} & =n^{2}(n-1)-2 n(n-1) \beta+2(n-\beta) \\
& =n\left(n^{2}-n+2\right)-2\left(n^{2}-n+1\right) \beta
\end{aligned}
$$

$$
\begin{aligned}
\{n+ & n-1) \beta\}\left\{6 n^{3}+4 n^{2}-41 n+40-n(2 n+1)^{2} \beta\right\} \\
= & n\left(6 n^{3}+4 n^{2}-41 n+40\right) \\
& +\left\{(n-1)\left(6 n^{3}+4 n^{2}-41 n+40\right)-n^{2}(2 n+1)^{2}\right\} \beta-2 n(2 n+1)^{2}(n-\beta) \\
= & \left(2 n^{4}+2 n^{3}-38 n^{2}+83 n-40\right) \beta-n\left(2 n^{3}+4 n^{2}+43 n-40\right) .
\end{aligned}
$$

Thus, combining these with the above inequality we obtain easily (12.1).

Q.E. D.

Next, we study the first factor of the second term of the right hand side of (11.8).

LEMMA 12.2. When $n \geqq 10$, we have

$$
\frac{x^{2} \sqrt{n-x F_{2}}(x)}{(1-x)^{5}}+\frac{X^{2} \sqrt{n-X} F_{2}(X)}{(X-1)^{5}}
$$




$$
\begin{aligned}
& >\frac{1}{X-1}\left\{\frac{32}{3} \cdot \frac{n(n-1)^{n+2}(n-4)^{2}\left(n^{2}-n+1\right) \sqrt{2 n^{2}-n+8}}{\sqrt{2 n+1}\left(8 n^{3}+18 n+1\right)^{2}}\right. \\
& +\frac{1}{96} \cdot \frac{n(n+2)^{n-2}(2 n-5)^{n-2}}{(n-1)^{5 / 2}(2 n+1)^{n}} \cdot\left(8 n^{3}-24 n^{2}-18 n+61\right) \\
& \left.\times\left(8 n^{4}-12 n^{3}-28 n^{2}+27 n+5\right)\right\} \quad \text { for } \quad \alpha_{1}(n) \leqq x<1 .
\end{aligned}
$$

Proof. By means of (7.6) and the inequality continued to it in the proof of Lemma 7.1, we obtain

$$
\begin{aligned}
\frac{x^{2} \sqrt{n-x} F_{2}(x)}{(1-x)^{5}}> & \frac{1}{t^{5}} \cdot \frac{4(n-4)^{2} \sqrt{2 n^{2}-n+8}}{(2 n+1)^{5 / 2}} \\
& \times \frac{8 B n(n-1)^{3}\left(n^{2}-n+1\right)(2 n+1)^{2} t^{4}}{3\left(8 n^{3}+18 n+1\right)^{2}} \\
= & \frac{32}{3} \cdot \frac{n(n-1)^{n+2}(n-4)^{2}\left(n^{2}-n+1\right) \sqrt{2 n^{2}-n+8}}{\sqrt{ } 2 n+1\left(8 n^{3}+18 n+1\right)^{2}} \cdot \frac{1}{t} \text { for } \alpha_{1}(n) \leqq x<1,
\end{aligned}
$$

where $t=1-x$, when $n \geqq 10$.

Since $x^{2} \sqrt{n-x}$ is increasing for $0<x<\frac{4}{5} n$ and $\frac{2 n+10}{2 n+1}<\frac{4}{5} n$ for $n \geqq 10$, we obtain analogously from (7.8) appeared in the proof of Lemma 7.2

$$
\begin{aligned}
\frac{X^{2} \sqrt{n-X} F_{2}(X)}{(X-1)^{5}}>\frac{1}{s^{5}} \cdot \sqrt{n-1} \cdot \frac{n(n+2)^{n-2}(2 n-5)^{n-2}}{96(n-1)^{3}(2 n+1)^{n}} \\
\quad \times\left(8 n^{3}-24 n^{2}-18 n+61\right)\left(8 n^{4}-12 n^{3}-28 n^{2}+27 n+5\right) s^{4} \\
=\frac{1}{96} \cdot \frac{n(n+2)^{n-2}(2 n-5)^{n-2}}{(n-1)^{5 / 2}(2 n+1)^{n}} \cdot\left(8 n^{3}-24 n^{2}-18 n+61\right) \\
\quad \times\left(8 n^{4}-12 n^{3}-28 n^{2}+27 n+5\right) \cdot \frac{1}{s} \quad \text { for } \quad 1<X<\frac{2 n+10}{2 n+1},
\end{aligned}
$$

where $s=X-1$, when $n \geqq 10$.

By Lemma 6.2, we have

$$
\frac{1}{t}=\frac{1}{1-x}>\frac{1}{X(x)-1}=\frac{1}{s} .
$$

Therefore, we obtain immediately (12.2) from the above inequalities (12.3) and (12.4).

Q.E. D.

Finally, from Lemma 12.1 and Lemma 12.2 we obtain the following

LEMMA 12.3. When $n \geqq 12$, we have the inequality

$$
\left\{\frac{x^{2} \sqrt{n-x} F_{2}(x)}{(1-x)^{5}}+\frac{X^{2} \sqrt{n-X} F_{2}(X)}{(X-1)^{5}}\right\} \cdot\{\tilde{\lambda}(X)-\tilde{\lambda}(\beta)\}>n \sqrt{n-1} B
$$




$$
\begin{aligned}
& \times\left[\frac{16}{3} \cdot \frac{(n-1)^{5 / 2}(n-4)^{2}\left(n^{2}-n+1\right) \sqrt{ } 2 n^{2}-n+8}{(2 n+1)^{3 / 2}\left(8 n^{3}+18 n+1\right)^{2}}+\frac{1}{192} \cdot \frac{(n+2)^{n-2}(2 n-5)^{n-2}}{(n-1)^{n+2}(2 n+1)^{n+1}} .\right. \\
& \left.\times\left(8 n^{3}-24 n^{2}-18 n+61\right)\left(8 n^{4}-12 n^{3}-28 n^{2}+27 n+5\right)\right] \\
& \times\left[\frac{\left(2 n^{4}+2 n^{3}-38 n^{2}+83 n-40\right) \beta-n\left(2 n^{3}+4 n^{2}+43 n-40\right)}{9\left\{n\left(n^{2}-n+2\right)-2\left(n^{2}-n+1\right) \beta\right\}}\right] \\
& \quad \text { for } \quad \alpha_{1}(n) \leqq x<1 .
\end{aligned}
$$

\section{$\S 13$. Some evaluations related with Lemma 12.3.}

LEMMA 13.1. We have the following inequalities

$$
\begin{gathered}
\frac{\left(2 n^{4}+2 n^{3}-38 n^{2}+83 n-40\right) \beta-n\left(2 n^{3}+4 n^{2}+43 n-40\right)}{(n-1)\left\{n\left(n^{2}-n+2\right)-2\left(n^{2}-n+1\right) \beta\right\}} \\
\geqq \frac{2(10103 \beta-13524)}{11(804-133 \beta)} \text { for } n \geqq 12, \\
\frac{4(16353 \beta-19728)}{15(1936-241 \beta)} \text { for } n \geqq 16,
\end{gathered}
$$

and

$$
\frac{105379 \beta-123246}{17(2772-307 \beta)} \quad \text { for } n \geqq 18 \text {. }
$$

Proof. Setting $u=\frac{1}{n}$, the left hand side of the above inequalities can be expressed as

$$
\Phi(u):=\frac{\left(2+2 u-38 u^{2}+83 u^{3}-40 u^{4}\right) \beta-\left(2+4 u+43 u^{2}-40 u^{3}\right)}{(1-u)\left\{1-u+2 u^{2}-2 u\left(1-u+u^{2}\right) \beta\right\}} .
$$

For the moment, we consider in this expression that $\beta$ is a constant such that $\sqrt{\frac{24}{13}}<\beta<\sqrt{2}$ and compute its logarithmic derivative with respect to $u$, we obtain

$$
\begin{aligned}
& \frac{\left(2-76 u+249 u^{2}-160 u^{3}\right) \beta-4-86 u+120 u^{2}}{\left(2+2 u-38 u^{2}+83 u^{3}-40 u^{4}\right) \beta-\left(2+4 u+43 u^{2}-40 u^{3}\right)}+\frac{1}{1-u} \\
& \quad+\frac{1-4 u+\left(2-4 u+6 u^{2}\right) \beta}{1-u+2 u^{2}-2 u\left(1-u+u^{2}\right) \beta} \\
& =\frac{\left(2-76 u+249 u^{2}-160 u^{3}\right) \beta-4-86 u+120 u^{2}}{\left(2+2 u-38 u^{2}+83 u^{3}-40 u^{4}\right) \beta-\left(2+4 u+43 u^{2}-40 u^{3}\right)} \\
& \quad+\frac{2-6 u+6 u^{2}+\left(2-8 u+12 u^{2}-8 u^{3}\right) \beta}{(1-u)\left\{1-u+2 u^{2}-2 u\left(1-u+u^{2}\right) \beta\right\}},
\end{aligned}
$$


for which, making the common denominator of the right hand side, we obtain its numarator as follows:

$$
\begin{aligned}
- & \left\{4+86 u-120 u^{2}-\left(2-76 u+249 u^{2}-160 u^{3}\right) \beta\right\}(1-u)\left\{1-u+2 u^{2}-2 u\left(1-u+u^{2}\right) \beta\right\} \\
+ & \left\{\left(2-2 u-38 u^{2}+83 u^{3}-40 u^{4}\right) \beta-\left(2+4 u+43 u^{2}-40 u^{3}\right)\right\} \\
& \times\left\{2-6 u+6 u^{2}+\left(2-8 u+12 u^{2}-8 u^{3}\right) \beta\right\}=-Q_{0}(u)+Q_{1}(u) \beta+Q_{2}(u) \beta^{2},
\end{aligned}
$$

where we set

$$
\left\{\begin{array}{l}
Q_{0}(u):=8+74 u-206 u^{2}+176 u^{3}-34 u^{4}, \\
Q_{1}(u):=2-72 u+409 u^{2}-660 u^{3}+425 u^{4}-68 u^{5}, \\
Q_{2}(u):=4-16 u+92 u^{2}-332 u^{3}+408 u^{4}-160 u^{5}-6 u^{6} .
\end{array}\right.
$$

We can easily show that $Q_{0}(u)$ is increasing and $Q_{1}(u)$ and $Q_{2}(u)$ are decreasing for $0 \leqq u \leqq \frac{1}{12}$, because

$$
\begin{aligned}
Q_{0}^{\prime}(u) & =74-412 u+528 u^{2}-136 u^{3}>74-\frac{412}{12}-\frac{136}{12^{3}}>0, \\
Q_{1}^{\prime}(u) & =-72+818 u-1980 u^{2}+1700 u^{3}-340 u^{4} \\
& <-72+\frac{818}{12}-\frac{1980}{12^{2}}+\frac{1700}{12^{3}}<-72+68.17-13.75+0.99<0,
\end{aligned}
$$

since $\frac{818}{1980 \times 2}=\frac{409}{1980}>\frac{1}{12}$, and

$$
\begin{aligned}
Q_{2}^{\prime}(u) & =-16+184 u-996 u^{2}+1632 u^{3}-800 u^{4}-36 u^{5} \\
& <-16+\frac{184}{12}-\frac{996}{12^{2}}+\frac{1632}{12^{3}}<-16+15.34-6.91+0.95<0,
\end{aligned}
$$

since $\frac{184}{996 \times 2}=\frac{23}{249}>\frac{1}{12}$. Hence we see that $-Q_{0}(u)+Q_{1}(u) \beta+Q_{2}(u) \beta^{2}$ is decreasing with respect to $u$ for $0 \leqq u \leqq \frac{1}{12}$, regarding as $\beta$ is a constant. On the other hand we have

$$
-Q_{0}(0)+Q_{1}(0) \beta+Q_{2}(0) \beta^{2}=-8+2 \beta+4 \beta^{2}>0
$$

since $\sqrt{\frac{24}{13}}<\beta<\sqrt{ } 2$, and

$$
\begin{aligned}
& -Q_{0}\left(\frac{1}{12}\right)+Q_{1}\left(\frac{1}{12}\right) \beta+Q_{2}\left(\frac{1}{12}\right) \beta^{2} \\
= & -\left(8+\frac{74}{12}-\frac{206}{12^{2}}+\frac{176}{12^{3}}-\frac{34}{12^{4}}\right)
\end{aligned}
$$




$$
\begin{aligned}
& +\left(2-\frac{72}{12}+\frac{409}{12^{2}}-\frac{660}{12^{3}}+\frac{425}{12^{4}}-\frac{68}{12^{5}}\right) \beta \\
& +\left(4-\frac{16}{12}+\frac{92}{12^{2}}-\frac{332}{12^{3}}+\frac{408}{12^{4}}-\frac{160}{12^{5}}-\frac{6}{12^{6}}\right) \beta^{2} \\
& <-\left(8+\frac{74}{12}-\frac{206}{12^{2}}+\frac{176}{12^{3}}-\frac{34}{12^{4}}\right)+\left(2-\frac{72}{12}+\frac{409}{12^{2}}-\frac{660}{12^{3}}+\frac{425}{12^{4}}\right) \beta \\
& +\left(4-\frac{16}{12}+\frac{92}{12^{2}}-\frac{332}{12^{3}}+\frac{408}{12^{4}}\right) \beta^{2}=\frac{1}{12^{4}} \cdot\left(-266174-31543 \beta+64968 \beta^{2}\right)<0 .
\end{aligned}
$$

Therefore, we can say that the original function $\Phi(u)$ is firstly increasing and then change to become decreasing at a point of the interval $0<u<\frac{1}{12}$.

Finally we shall compute the values of $\Phi(u)$ at $u=\frac{1}{12}, \frac{1}{16}$ and $\frac{1}{18}$, and compare them with the one at $u=0$. Since we have

$$
\begin{aligned}
& 2 \cdot 12^{4}+2 \cdot 12^{3}-38 \cdot 12^{2}+83 \cdot 12-40=40412=4 \cdot 10103, \\
& 2 \cdot 16^{4}+2 \cdot 16^{3}-38 \cdot 16^{2}+83 \cdot 16-40=130824=8 \cdot 16353, \\
& 2 \cdot 18^{4}+2 \cdot 18^{3}-38 \cdot 18^{2}+83 \cdot 18-40=210758=2 \cdot 105379
\end{aligned}
$$

and

$$
\begin{aligned}
& 2 \cdot 12^{3}+4 \cdot 12^{2}+43 \cdot 12-40=4508, \\
& 2 \cdot 16^{3}+4 \cdot 16^{2}+43 \cdot 16-40=9864, \\
& 2 \cdot 18^{3}+4 \cdot 18^{2}+43 \cdot 18-40=13694,
\end{aligned}
$$

we obtain

$$
\begin{aligned}
& \Phi\left(\frac{1}{12}\right)=\frac{4 \cdot 10103 \beta-12 \cdot 4508}{11(12 \cdot 134-2 \cdot 133 \beta)}=\frac{2(10103 \beta-13524)}{11(804-133 \beta)}, \\
& \Phi\left(\frac{1}{16}\right)=\frac{8 \cdot 16353 \beta-16 \cdot 9864}{15(16 \cdot 242-2 \cdot 241 \beta)}=\frac{4(16353 \beta-19728)}{15(1936-241 \beta)}, \\
& \Phi\left(\frac{1}{18}\right)=\frac{2 \cdot 105379 \beta-18 \cdot 13694}{17(18 \cdot 308-2 \cdot 307 \beta)}=\frac{105379 \beta-123246}{17(2772-307 \beta)} .
\end{aligned}
$$

We see easily $\Phi\left(\frac{1}{18}\right)<\Phi(0)=2(\beta-1)$, because

$$
105379 \beta-123246<34(\beta-1)(2772-307 \beta),
$$

which is equivalent to

$$
10438 \beta^{2}+693 \beta-28998<0 .
$$

In fact we have 
$10438 \beta^{2}+693 \beta-28998<10438 \cdot 2+693 \cdot \sqrt{ } 2-28998=-8122+693 \cdot \sqrt{2}<0$ for $\sqrt{\frac{24}{13}}<\beta<\sqrt{2}$. Thus, we see that

$$
\Phi(0)>\Phi\left(\frac{1}{18}\right)>\Phi\left(\frac{1}{16}\right)>\Phi\left(\frac{1}{12}\right) .
$$

From these things we obtain easily (13.1), (13.2) and (13.3).

Q.E. D.

LEMMA 13.2. We have the following inequalities

$$
\begin{gathered}
\frac{16}{3} \cdot \frac{(n-1)^{7 / 2}(n-4)^{2}\left(n^{2}-n+1\right) \sqrt{2 n^{2}-n+8}}{n(2 n+1)^{3 / 2}\left(8 n^{3}+18 n+1\right)^{2}} \geqq \\
\quad \frac{2^{9} \cdot(11)^{7 / 2} \cdot(71)^{1 / 2} \cdot 133}{3^{2} \cdot 5^{3} \cdot(14041)^{2}} \text { for } n \geqq 12, \\
\quad \frac{2^{11 / 2} \cdot 3^{2} \cdot 5^{7 / 2} \cdot 7^{1 / 2} \cdot 241}{(11)^{3 / 2} \cdot(11019)^{2}} \quad \text { for } n \geqq 16,
\end{gathered}
$$

and

$$
\frac{2^{11 / 2} \cdot 7^{2} \cdot(17)^{7 / 2} \cdot 307 \cdot(319)^{1 / 2}}{3^{3} \cdot(37)^{3 / 2} \cdot(46981)^{2}} \quad \text { for } n \geqq 18 .
$$

Proof. Setting $u=\frac{1}{n}$, the left hand side of the above inequalities can be expressed as

$$
\frac{16}{3} \cdot \frac{(1-u)^{7 / 2}(1-4 u)^{2}\left(1-u+u^{2}\right) \sqrt{ } 2-u+8 u^{2}}{(2+u)^{3 / 2}\left(8+18 u^{2}+u^{3}\right)^{2}}
$$

First, we show that $(1-4 u)^{4}\left(2-u+8 u^{2}\right)$ is decreasing for $0 \leqq u \leqq \frac{1}{12}$, because

$$
\left\{(1-4 u)^{4}\left(2-u+8 u^{2}\right)\right\}^{\prime}=-(1-4 u)^{3}\left(33-36 u+192 u^{2}\right)<0 .
$$

Hence, putting $n=12,16$ and 18 in the expression above of $n$, we obtain these inequalities.

Q.E. D.

$$
\text { LEMMA 13.3. Setting } u=\frac{1}{n} \text {, we have }
$$

$$
\begin{aligned}
\frac{1}{192} & \frac{(n+2)^{n-2}(2 n-5)^{n-2}}{n(n-1)^{n+1}(2 n+1)^{n+1}} \cdot\left(8 n^{3}-24 n^{2}-18 n+61\right)\left(8 n^{4}-12 n^{3}-28 n^{2}+27 n+5\right) \\
= & \frac{1}{2^{17 / 2} \cdot 3} \cdot \frac{\sqrt{ } 2-5 u}{(1-u)^{2}(1+2 u)^{2}(2+u)} \cdot \frac{e(n-1)(e(n / 2))^{2}}{\sqrt{e(2 n)(e((2 n-5) / 5))^{5 / 2}}} \\
& \times\left(8-24 u-18 u^{2}+61 u^{3}\right)\left(8-12 u-28 u^{2}+27 u^{3}+5 u^{4}\right) .
\end{aligned}
$$

Proof. The left hand side of (13.9) can be written as 


$$
\frac{1}{2^{6} \cdot 3} \cdot \frac{(1+2 u)^{n-2}(2-5 u)^{n-2}}{(1-u)^{n+1}(2+u)^{n+1}} \cdot\left(8-24 u-18 u^{2}+61 u^{3}\right)\left(8-12 u-28 u^{2}+27 u^{3}+5 u^{4}\right) \text {. }
$$

Using the notation $e(n)=e_{n}=\left(1+\frac{1}{n}\right)^{n}$ introduced in $\S 10$, we have

$$
\begin{aligned}
& (1-u)^{n+1}=(1-u)^{2} / e(n-1), \quad(2+u)^{n+1}=2^{n} \cdot(2+u) \cdot \sqrt{e(2 n)}, \\
& (1+2 u)^{n-2}=\frac{1}{(1+2 u)^{2}} \cdot(e(n / 2))^{2}, \quad(2-5 u)^{n-2}=2^{n-5 / 2} \cdot \sqrt{2-5 u} /\left(e\left(\frac{2 n-5}{5}\right)\right)^{5 / 2} .
\end{aligned}
$$

Putting these into the above expression, we can obtain easily the right hand side of (13.9).

Q.E.D.

LEMMA 13.4. The function $e(n-1)(e(n / 2))^{2} / \sqrt{e(2 n)}(e((2 n-5) / 5))^{5 / 2}$ of $n$ is decreasing for $n>5 / 2$.

Proof. From the definition of $e(n)$, we obtain

$$
\begin{aligned}
& \quad \frac{d}{d n}\left\{(n-1) \log \left(1+\frac{1}{n-1}\right)+n \log \left(1+\frac{2}{n}\right)-n \log \left(1+\frac{1}{2 n}\right)\right. \\
& \left.-\left(n-\frac{5}{2}\right) \log \left(1+\frac{5}{2 n-5}\right)\right\}=\log \left(1+\frac{1}{n-1}\right)+\log \left(1+-\frac{2}{n}\right)-\log \left(1+-\frac{1}{2 n}\right) \\
& -\log \left(1+\frac{5}{2 n-5}\right)-(n-1) \frac{1 /(n-1)^{2}}{1+(1 /(n-1))}-n \frac{2 / n^{2}}{1+(2 / n)}+n \frac{1 / 2 n^{2}}{1+(1 / 2 n)} \\
& +\left(n-\frac{5}{2}\right) \frac{10 /(2 n-5)^{2}}{1+(5 /(2 n-5))}=\log \frac{(n+2)(2 n-5)}{(n-1)(2 n+1)}-\frac{1}{n}-\frac{2}{n+2}+\frac{1}{2 n+1}+\frac{5}{2 n} \\
& =\log \frac{2 n^{2}-n-10}{2 n^{2}-n-1}+\frac{3(5 n+2)}{2 n(n+2)(2 n+1)} .
\end{aligned}
$$

Setting $u=\frac{1}{n}$, the above expression can be written as

$$
\frac{3}{2} \cdot \frac{u^{2}(5+2 u)}{(1+2 u)(2+u)}+\log \frac{2-u-10 u^{2}}{2-u-u^{2}}
$$

which becomes 0 at $u=0$. Its derivative with respect to $u$ is

$$
\begin{aligned}
& -\frac{3 u\left(20+37 u+20 u^{2}+4 u^{3}\right)}{2(1+2 u)^{2}(2+u)^{2}}-\frac{1+20 u}{2-u-10 u^{2}}+\frac{1+2 u}{2-u-u^{2}} \\
= & \frac{3 u\left(20+37 u+20 u^{2}+4 u^{3}\right)}{2(1+2 u)^{2}(2+u)^{2}}-\frac{9 u(4-u)}{\left(2-u-10 u^{2}\right)\left(2-u-u^{2}\right)} \\
= & -\frac{3 u\left(16+388 u+1160 u^{2}+903 u^{3}-195 u^{4}-530 u^{5}-244 u^{6}-40 u^{7}\right)}{2(1+2 u)^{2}(2+u)^{2}\left(2-u-10 u^{2}\right)\left(2-u-u^{2}\right)}<0
\end{aligned}
$$

for $0<u<2 / 5$ and hence we see that 
466

TOMINOSUKE OTSUKI

$$
\log \frac{2 n^{2}-n-10}{2 n^{2}-n-1}+\frac{3(5 n+2)}{2 n(n+2)(2 n+1)}<0 \text { for } n>\frac{5}{2} \text {, }
$$

which implies the statement of this lemma.

Q.E.D.

LEMMA 13.5. We have the following inequalities

$$
\frac{1}{192} \cdot \frac{(n+2)^{n-2}(2 n-5)^{n-2}}{n(n-1)^{n+1}(2 n+1)^{n+1}} \cdot\left(8 n^{3}-24 n^{2}-18 n+61\right)\left(8 n^{4}-12 n^{3}-28 n^{2}+27 n+5\right) \geqq
$$

$$
\begin{aligned}
& \frac{\sqrt{19} \cdot 167 \cdot 1459}{2^{31 / 2} \cdot 3^{7 / 2} \cdot 5^{2}} \cdot \frac{e_{15} \cdot\left(e_{8}\right)^{2}}{\sqrt{e_{32}} \cdot\left(e_{5.4}\right)^{5 / 2}} \quad \text { for } 12 \leqq n<16, \\
& \frac{2933 \cdot 10409}{2^{41 / 2} \cdot 3^{5 / 2} \cdot 5 \cdot 11} \cdot \frac{e_{17} \cdot\left(e_{9}\right)^{2}}{\sqrt{e_{36}} \cdot\left(e_{6.2}\right)^{5 / 2}} \quad \text { for } 16 \leqq n<18,
\end{aligned}
$$

and

$$
\frac{\sqrt{31} \cdot 38617 \cdot 761243}{2^{15} \cdot 3^{6} \cdot 5^{2} \cdot 17^{2} \cdot 37} \quad \text { for } n \geqq 18 \text {. }
$$

Proof. Regarding the right hand side of (13.9), we see that the function $\frac{\sqrt{2-5 u}}{(1-u)^{2}(1+2 u)^{2}(2+u)}$ is decreasing for $0<u \leqq \frac{1}{12}$, because

$$
\begin{aligned}
& \frac{d}{d u} \log -\frac{\sqrt{2-5 u}}{(1-u)^{2}(1+2 u)^{2}(2+u)}=-\frac{5}{2(2-5 u)}+\frac{2}{1-u}-\frac{4}{1+2 u}-\frac{1}{2+u} \\
& =-\frac{5}{2(2-5 u)}-\frac{1}{2+u}-\frac{2(1-4 u)}{(1-u)(1+2 u)}<0,
\end{aligned}
$$

and both of $8-24 u-18 u^{2}+61 u^{3}$ and $8-12 u-28 u^{2}+27 u^{3}+5 u^{4}$ are clearly decreasing for $0<u \leqq \frac{1}{12}$.

Now, putting $u=\frac{1}{12}, \frac{1}{16}, \frac{1}{18}$ in the expression

$$
\frac{1}{2^{17 / 2} \cdot 3} \cdot \frac{\sqrt{2-5 u}}{(1-u)^{2}(1+2 u)^{2}(2+u)} \cdot\left(8-24 u-18 u^{2}+61 u^{3}\right)\left(8-12 u-28 u^{2}+27 u^{3}+5 u^{4}\right)
$$

we obtain respectively:

$$
\begin{aligned}
& \frac{1}{2^{17 / 2} \cdot 3} \cdot \frac{(12)^{9 / 2} \cdot \sqrt{19}}{(11)^{2} \cdot(14)^{2} \cdot 25} \cdot \frac{8 \cdot(12)^{3}-24 \cdot(12)^{2}-18 \cdot 12+61}{(12)^{3}} \\
& \quad \times \frac{8 \cdot(12)^{4}-12(12)^{3}-28 \cdot(12)^{2}+27 \cdot 12+5}{(12)^{4}} \\
& \quad=\frac{\sqrt{19 \cdot 10213 \cdot 141449}}{2^{17 / 2} \cdot 3 \cdot(11)^{2} \cdot(14)^{2} \cdot 25 \cdot(12)^{5 / 2}}=\frac{\sqrt{19 \cdot 167 \cdot 1459}}{2^{31 / 2} \cdot 3^{7 / 2} \cdot 5^{2}}
\end{aligned}
$$




$$
\begin{aligned}
& \frac{1}{2^{17 / 2} \cdot 3} \cdot \frac{(16)^{9 / 2} \cdot \sqrt{27}}{(15)^{2} \cdot(18)^{2} \cdot 33} \cdot \frac{8 \cdot(16)^{3}-24 \cdot(16)^{2}-18 \cdot 16+61}{(16)^{3}}-16 \\
& \quad \times \frac{8 \cdot(16)^{4}-12 \cdot(16)^{3}-28 \cdot(16)^{2}+27 \cdot 16+5}{(16)^{4}} \\
& \quad=\frac{3^{3 / 2} \cdot 26397 \cdot 468405}{2^{17 / 2} \cdot 3 \cdot(15)^{2} \cdot(18)^{2} \cdot 33 \cdot(16)^{5 / 2}}=\frac{2933 \cdot 10409}{2^{41 / 2} \cdot 3^{5 / 2} \cdot 5 \cdot 11}
\end{aligned}
$$

and

$$
\begin{aligned}
& \frac{1}{2^{17 / 2} \cdot 3} \cdot \frac{(18)^{9 / 2} \cdot \sqrt{ } 31}{(17)^{2} \cdot(20)^{2} \cdot 37} \cdot \frac{8 \cdot(18)^{3}-24 \cdot(18)^{2}-18 \cdot 18+61}{(18)^{3}} \\
& \quad \times \frac{8 \cdot(18)^{4}-12 \cdot(18)^{3}-28 \cdot(18)^{2}+27 \cdot 18+5}{(18)^{4}} \\
& \quad=\frac{\sqrt{31 \cdot 38617 \cdot 761243}}{2^{17 / 2} \cdot 3 \cdot(17)^{2} \cdot(20)^{2} \cdot 37 \cdot(18)^{5 / 2}}=\frac{\sqrt{31} \cdot 38617 \cdot 761243}{2^{15} \cdot 3^{6} \cdot 5^{2} \cdot(17)^{2} \cdot 37}
\end{aligned}
$$

From these values and Lemma 13.4, we obtain easily (13.10), (13.11) and (13.12). Q.E.D.

$\S 14$. Fine evaluations of $V(x, X(x))$.

For the present purpose, we shall do more fine evaluations of the function $V(x, X(x))$ for $\alpha_{1}(n) \leqq x<1$ than the ones in $\S 9$ and $\S 10$.

LEMMA 14.1. We have the following inequalities

$$
\begin{aligned}
\frac{112}{27} & \cdot \frac{(n-1)^{5 / 2}(n-4)^{2}\left(n^{2}-n+1\right)(2 n+1)^{3 / 2}\left(2 n^{2}-n+8\right)^{1 / 2}}{\left(8 n^{3}+18 n+1\right)^{2}} \\
\times & {\left[\frac{1}{\left(2 n^{2}-n+8\right)^{2}}+\frac{4 n^{2}+9 n-10}{4(n-1)(n+5)^{2}\left(2 n^{2}-n-10\right)}\right]>} \\
(14.1) \quad & \frac{2^{8} \cdot(11)^{4} \cdot(71)^{1 / 2} \cdot 133}{3^{7} \cdot 7 \cdot(14041)^{2}}+\frac{2^{2} \cdot 3^{3} \cdot 7 \cdot(11)^{3} \cdot(71)^{1 / 2} \cdot 193}{(17)^{2} \cdot(14041)^{2}} \text { for } 12 \leqq n<16, \\
\text { (14.2) } \quad & \frac{2^{3} \cdot 3^{7 / 2} \cdot 5^{5 / 2} \cdot 7^{3 / 2} \cdot(37)^{3 / 2} \cdot 241-2^{20} \cdot 5^{3 / 2} \cdot(37)^{3 / 2} \cdot 181 \cdot 241}{(319)^{2} \cdot(11019)^{2}}
\end{aligned}
$$

and

$$
\frac{2^{19 / 2} \cdot 3^{4} \cdot 7^{3} \cdot(17)^{3 / 2} \cdot 307 \cdot(319)^{1 / 2}}{5 \cdot(23)^{2} \cdot 31 \cdot(46981)^{2}} \quad \text { for } n \geqq 18
$$

Proof. Setting $u=\frac{1}{n}$, the left hand side can be written as in Lemma 10.1:

$$
\frac{2^{4} \cdot 7}{3^{3}} \cdot \frac{(1-u)^{5 / 2}(1-4 u)^{2}\left(1-u+u^{2}\right)(2+u)^{3 / 2}\left(2-u+8 u^{2}\right)^{1 / 2}}{\left(8+18 u^{2}+u^{3}\right)^{2}}
$$




$$
\times\left[\frac{u}{\left(2-u+8 u^{2}\right)^{2}}+\frac{4+9 u-10 u^{2}}{4(1-u)(1+5 u)^{2}\left(2-u-10 u^{2}\right)}\right] .
$$

We see that $(1-4 u)^{2}\left(2-u+8 u^{2}\right)^{1 / 2}$ is decreasing for $0<u<\frac{1}{4}$, because its logarithmic derivative is

$$
-\frac{8}{1-4 u}-\frac{1-16 u}{2\left(2-u+8 u^{2}\right)}=-\frac{33-36 u+192 u^{2}}{2(1-4 u)\left(2-u+8 u^{2}\right)}<0
$$

and $\frac{u}{\left(2-u+8 u^{2}\right)^{2}}$ is increasing for $0<u<\frac{1}{4}$, because its logarithmic derivative is

$$
\frac{1}{u}+\frac{2(1-16 u)}{2-u+8 u^{2}}=\frac{2+u-24 u^{2}}{u\left(2-u+8 u^{2}\right)}>0 .
$$

$4+9 u-10 u^{2}$ is also increasing for $0<u<\frac{1}{4}$, We showed in the proof of Lemma 10.1 that $(1-u)(1+5 u)^{2}\left(2-u-10 u^{2}\right)$ is increasing for $0<u \leqq \frac{1}{10}$. Making use of these facts, we can do the following evaluations.

When $12 \leqq n<16\left(\frac{1}{16}<u \leqq \frac{1}{12}\right)$, we have

(the left hand side of $(10.1))>$

$$
\begin{aligned}
\frac{2^{4} \cdot 7}{3^{3}} & \cdot \frac{(11 / 12)^{5 / 2} \cdot(2 / 3)^{2} \cdot 133 /(12)^{2} \cdot(33 / 16)^{3 / 2} \cdot(284)^{1 / 2} / 12 \cdot(12)^{6}}{(14041)^{2}} \\
& \times\left[\frac{1 / 16 \cdot(16)^{4}}{(504)^{2}}+\frac{1158 /(16)^{2} \cdot(12)^{5}}{4 \cdot 11 \cdot(17)^{2} \cdot 266}\right] \\
= & \frac{2^{2} \cdot 7 \cdot(11)^{4} \cdot(71)^{1 / 2} \cdot 133}{3^{3} \cdot(14041)^{2}} \cdot\left[\frac{2^{6}}{3^{4} \cdot 7^{2}}+\frac{3^{6} \cdot 193}{11 \cdot(17)^{2} \cdot 133}\right] \\
= & \frac{2^{8} \cdot(11)^{4} \cdot(71)^{1 / 2} \cdot 133}{3^{7} \cdot 7 \cdot(14041)^{2}}+\frac{2^{2} \cdot 3^{3} \cdot 7 \cdot(11)^{3} \cdot(71)^{1 / 2} \cdot 193}{(17)^{2} \cdot(14041)^{2}} .
\end{aligned}
$$

When $16 \leqq n<18\left(\frac{1}{18}<u \leqq \frac{1}{16}\right)$, we have

(the left hand side of (10.1)) >

$$
\begin{aligned}
\frac{2^{4} \cdot 7}{3^{3}} & \cdot \frac{(15 / 16)^{5 / 2} \cdot(3 / 4)^{2} \cdot 241 /(16)^{2} \cdot(37 / 18)^{3 / 2} \cdot(504)^{1 / 2} / 16 \cdot(16)^{6}}{(33057)^{2}} \\
& \times\left[\frac{1 / 18 \cdot(18)^{4}}{(638)^{2}}+\frac{1448 /(18)^{2} \cdot(16)^{5}}{4 \cdot 15 \cdot(21)^{2} \cdot 486}\right] \\
= & \frac{2^{2} \cdot 5^{5 / 2} \cdot 7^{3 / 2} \cdot(37)^{3 / 2} \cdot 241}{3^{5 / 2} \cdot(11019)^{2}} \cdot\left[\frac{2 \cdot 3^{6}}{(319)^{2}}+\frac{2^{18} \cdot 181}{3^{12} \cdot 5 \cdot 7^{2}}\right]
\end{aligned}
$$




$$
=\frac{2^{3} \cdot 3^{7 / 2} \cdot 5^{5 / 2} \cdot 7^{3 / 2} \cdot(37)^{3 / 2} \cdot 241}{(319)^{2} \cdot(11019)^{2}}+\frac{2^{20} \cdot 5^{3 / 2} \cdot(37)^{3 / 2} \cdot 181 \cdot 241}{3^{29 / 2} \cdot 7^{1 / 2} \cdot(11019)^{2}} .
$$

When $18 \leqq n\left(0<u \leqq \frac{1}{18}\right)$, we have

(the left hand side of $(10.1))>$

$$
\begin{aligned}
\frac{2^{4} \cdot 7}{3^{3}} & \cdot \frac{(17 / 18)^{5 / 2} \cdot(7 / 9)^{2} \cdot 307 /(18)^{2} \cdot 2^{3 / 2} \cdot(638)^{1 / 2} / 18 \cdot(18)^{6}}{(46981)^{2}} \\
& \times\left[0+\frac{4 \cdot(18)^{5}}{4 \cdot 17 \cdot(23)^{2} \cdot 620}\right]=\frac{2^{19 / 2} \cdot 3^{4} \cdot 7^{3} \cdot(17)^{3 / 2} \cdot 307 \cdot(319)^{1 / 2}}{5 \cdot(23)^{2} \cdot 31 \cdot(46981)^{2}} \cdot \quad \text { Q. E. D. }
\end{aligned}
$$

LEMMA 14.2. We have the following inequalities

$$
\frac{1}{96} \cdot \frac{n(n+2)^{n-2}(2 n-5)^{n-2}}{(n-1)^{n+4}(2 n+1)^{n}} \cdot\left(8 n^{3}-24 n^{2}-18 n+61\right)\left(8 n^{4}-12 n^{3}-28 n^{2}+27 n+5\right)>
$$

$$
\begin{aligned}
& \frac{(19)^{1 / 2} \cdot 10213 \cdot 141449}{2^{21 / 2} \cdot 3^{3 / 2} \cdot 7^{2} \cdot(11)^{5}} \cdot \frac{e_{15} \cdot\left(e_{8}\right)^{2}}{\sqrt{e_{32} \cdot\left(e_{5.4}\right)^{5 / 2}}} \quad \text { for } 12 \leqq n<16, \\
& \frac{2933 \cdot 10409}{2^{23 / 2} \cdot 3^{9 / 2} \cdot 5^{4}} \cdot \frac{e_{17} \cdot\left(e_{9}\right)^{2}}{\sqrt{e_{36} \cdot\left(e_{6.2}\right)^{5 / 2}}} \quad \text { for } \quad 16 \leqq n<18
\end{aligned}
$$

and

$$
\frac{7 \cdot 38617 \cdot 761243}{2^{29 / 2} \cdot 3^{15} \cdot 5} \quad \text { for } n \geqq 18
$$

Proof. Setting $u=\frac{1}{n}$, the left hand side can be written as (10.3):

$$
\begin{aligned}
& \frac{1}{2^{15 / 2} \cdot 3} \cdot \frac{\sqrt{ } 2-5 u}{(1-u)^{5}(1+2 u)^{2}} \cdot \frac{e(n-1)(e(n / 2))^{2}}{\sqrt{ } e(2 n)(e((2 n-5) / 5))^{5 / 2}} \\
& \quad \times\left(8-24 u-18 u^{2}+61 u^{3}\right)\left(8-12 u-28 u^{2}+27 u^{3}+5 u^{4}\right) .
\end{aligned}
$$

We showed that $\frac{\sqrt{ } 2-5 u}{(1-u)^{5}(1+2 u)^{2}}$ is decreasing for $0<u<\frac{41-\sqrt{ } 1161}{2 \cdot 130}=\frac{1}{37.53 \cdots}$ and increasing for $\frac{41-\sqrt{1161}}{2 \cdot 130}<u<\frac{41+\sqrt{ } 1160}{2 \cdot 130}=\frac{1}{3.46 \ldots}$ and its value at $u=$ $\frac{41-\sqrt{1161}}{2 \cdot 130}$ is $1.4095 \cdots(>7 / 5)$, and that the both functions $8-24 u-18 u^{2}+61 u^{3}$ and $8-12 u-28 u^{2}+27 u^{3}+5 u^{4}$ are decreasing for $0<u \leqq(1 / 10)$. By means of these facts and Lemma 13.4 we can do the following evaluations.

When $12 \leqq n<16\left(\frac{1}{16}<u \leqq \frac{1}{12}\right)$, we have

(the left hand side of $(10.2))>$

$$
\frac{1}{2^{15 / 2} \cdot 3} \cdot \frac{\sqrt{19} \cdot(12)^{13 / 2}}{(11)^{5} \cdot(14)^{2}} \cdot \frac{e_{15} \cdot\left(e_{8}\right)^{2}}{\sqrt{e_{32} \cdot\left(e_{5.4}\right)^{5 / 2}}} \cdot \frac{1}{(12)^{7}} \cdot 10213 \cdot 141449
$$




$$
=\frac{(19)^{1 / 2} \cdot 10213 \cdot 141449}{2^{21 / 2} \cdot 3^{3 / 2} \cdot 7^{2} \cdot(11)^{5}} \cdot \frac{e_{15} \cdot\left(e_{8}\right)^{2}}{\sqrt{e_{32}} \cdot\left(e_{5.4}\right)^{5 / 2}} .
$$

When $16 \leqq n<18\left(\frac{1}{18}<u \leqq \frac{1}{16}\right)$, we have

(the left hand side of $(10.2))>$

$$
\begin{aligned}
& \frac{1}{2^{15 / 2} \cdot 3} \cdot \frac{\sqrt{27} \cdot(16)^{13 / 2}}{(15)^{5} \cdot(18)^{2}} \cdot \frac{e_{17} \cdot\left(e_{9}\right)^{2}}{\sqrt{e_{36}} \cdot\left(e_{6.2}\right)^{5 / 2}} \\
& \quad \times \frac{1}{(16)^{7}} \cdot 26397 \cdot 468405=\frac{2933 \cdot 10409}{2^{23 / 2} \cdot 3^{9 / 2} \cdot 5^{4}} \cdot \frac{e_{17}}{\sqrt{e_{36}} \cdot\left(e_{9}\right)^{2}} \cdot .\left(e_{6.2}\right)^{5 / 2}
\end{aligned}
$$

When $18 \leqq n\left(0<u \leqq \frac{1}{18}\right)$, we have

(the left hand side of $(10.2))>$

$$
\frac{1}{2^{15 / 2} \cdot 3} \cdot \frac{7}{5} \cdot 1 \cdot \frac{1}{(18)^{7}} \cdot 38617 \cdot 761243=\frac{7 \cdot 38617 \cdot 761243}{2^{29 / 2} \cdot 3^{15} \cdot 5}
$$

LEMMA 14.3. The function $e((n-1) / 3) / e((2 n-5) / 6)$ of $n$ is decreasing for $n>5 / 2$

Proof. From the definition of $e(n)$, we have

$$
e\left(\frac{n-1}{3}\right)=\left(\frac{n+2}{n-1}\right)^{(n-1) / 3} \text { and } e\left(\frac{2 n-5}{6}\right)=\left(\frac{2 n+1}{2 n-5}\right)^{(2 n-5) / 6}
$$

and hence

$$
\begin{aligned}
\frac{d}{d n}[ & \left.\frac{n-1}{3} \log \frac{n+2}{n-1}-\frac{2 n-5}{6} \log \frac{2 n+1}{2 n-5}\right] \\
= & \frac{1}{3}\left(\log \frac{n+2}{n-1}-\log \frac{2 n+1}{2 n-5}\right)+\frac{n-1}{3} \cdot \frac{-3}{(n+2)(n-1)} \\
& \quad-\frac{2 n-5}{6} \cdot \frac{-12}{(2 n+1)(2 n-5)} \\
= & \frac{1}{3} \log \frac{2 n^{2}-n-10}{2 n^{2}-n-1}+\frac{3}{(n+2)(2 n+1)} .
\end{aligned}
$$

Setting $u=1 / n$, the above expression can be written as

$$
\frac{3 u^{2}}{(1+2 u)(2+u)}+\frac{1}{3} \log \frac{2-u-10 u^{2}}{2-u-u^{2}},
$$

which becomes 0 at $u=0$. Its derivative with respect to $u$ is

$$
\frac{3 u(4+5 u)}{(1+2 u)^{2}(2+u)^{2}}-\frac{3 u(4-u)}{\left(2-u-10 u^{2}\right)\left(2-u-u^{2}\right)}
$$




$$
=-\frac{27 u\left(8+24 u+12 u^{2}-11 u^{3}-6 u^{4}\right)}{(1+2 u)(2+u)^{2}\left(2-u-10 u^{2}\right)\left(2-u-u^{2}\right)}<0 \quad \text { for } \quad 0<u<\frac{2}{5}
$$

and hence we see that

$$
\frac{1}{3} \log \frac{2 n^{2}-n-10}{2 n^{2}-n-1}+\frac{3}{(n+2)(2 n+1)}<0 \quad \text { for } n>\frac{5}{2},
$$

which implies the statement of this lemma.

Q.E.D.

LEMMA 14.4. We have the following inequalities

$$
\begin{aligned}
& \frac{n}{12(n-1)^{n-3 / 2}} \cdot \frac{1}{(2 n+1)^{n-3 / 2}(n+2)^{3 / 2}(2 n-5)^{3 / 2}\left(4 n^{2}-2 n+7\right)^{2}} \\
& \quad \times\left[4 n ( n - 1 ) ^ { n - 4 } ( n + 5 ) ( 2 n + 1 ) ^ { n - 3 } ( 4 n ^ { 2 } - 2 n + 7 ) ^ { 2 } \left(48 n^{4}-52 n^{3}+342 n^{2}\right.\right. \\
& \quad-198 n+616)-3(n-4)(n+2)^{n-2}(2 n-5)^{n-2}\left(256 n^{6}-992 n^{5}+80 n^{4}\right. \\
& \left.\quad+4120 n^{3}-3592 n^{2}-5474 n+3010\right]< \\
& \frac{2^{19 / 2} \cdot 3 \cdot 17 \cdot 1489}{5^{2} \cdot 7^{3 / 2} \cdot(11)^{5 / 2} \cdot(19)^{3 / 2}}-\frac{2^{19 / 2} \cdot(11)^{1 / 2} \cdot 13836023}{3^{7} \cdot 5^{2} \cdot 7^{5 / 2} \cdot(111)^{2}} \cdot\left(\frac{e_{5}}{e_{4.5}}\right)^{3} \text { for } 12 \leqq n<16, \\
& \frac{2^{19 / 2} \cdot 7 \cdot 4657}{3^{15 / 2} \cdot 5^{5 / 2} \cdot(11)^{3 / 2}}-\frac{3^{15 / 2} \cdot 5^{1 / 2} \cdot 748947}{2^{19 / 2} \cdot 7^{2} \cdot 11 \cdot(181)^{2}} \cdot\left(\frac{e_{17 / 3}}{e_{31 / 6}}\right)^{3} \quad \text { for } \quad 16 \leqq n<18
\end{aligned}
$$

and

$$
\frac{2 \cdot 3^{3} \cdot 23 \cdot 1210861}{5^{3 / 2} \cdot(17)^{5 / 2} \cdot(31)^{3 / 2} \cdot(37)^{3 / 2}}-\frac{7 \cdot(17)^{1 / 2} \cdot 3431922239}{2^{12} \cdot 3^{6} \cdot 5^{5 / 2} \cdot 31 \cdot 37} \quad \text { for } n \geqq 18
$$

Proof. Setting $u=1 / n$, the left hand side can be written as (10.7):

$$
\begin{aligned}
& \frac{(1+5 u)\left(48-52 u+342 u^{2}-198 u^{3}+616 u^{4}\right)}{3(1+2 u)^{3 / 2}(2-5 u)^{3 / 2}(1-u)^{5 / 2}(2+u)^{3 / 2}} \\
& \quad-\frac{(1-4 u) \sqrt{1-u}}{4(1+2 u)^{5 / 2}(2-5 u)(2+u)} \cdot \frac{1}{\left(4-2 u+7 u^{2}\right)^{2}} \cdot\left(e\left(\frac{n-1}{3}\right) / e\left(-\frac{2 n-5}{6}\right)\right)^{3} \\
& \quad \times\left(256-992 u+80 u^{2}+4120 u^{3}-3592 u^{4}-5474 u^{5}+3010 u^{6}\right) .
\end{aligned}
$$

We showed in the proof of Lemma 10.3 that the functions of $u$ :

$$
\begin{array}{ll}
(1+5 u)\left(48-52 u+342 u^{2}-198 u^{3}+616 u^{4}\right) & \text { increasing, } \\
(1+2 u)^{3}(2-5 u)^{3}(1-u)^{5}(2+u)^{3} & \text { decreasing, } \\
(1-4 u) \sqrt{1-u} & \text { decreasing, } \\
(1+2 u)^{5 / 2}(2-5 u)(2+u) & \text { increasing, } \\
256-992 u+80 u^{2}+4120 u^{3}-3592 u^{4}-5474 u^{5}+3010 u^{6} & \text { decreasing }
\end{array}
$$

for $0<u \leqq(1 / 10)$. By means of these facts and Lemma 14.3 we can do the following evaluations. 
When $12 \leqq n<16\left(\frac{1}{16}<u \leqq \frac{1}{12}\right)$, we have

(the left hand side of $(10.6))<$

$$
\begin{aligned}
\frac{17 \cdot 952960 \cdot(12)^{7}}{3 \cdot(12)^{5} \cdot(14)^{3 / 2} \cdot(19)^{3 / 2} \cdot(11)^{5 / 2} \cdot(25)^{3 / 2}} \\
\quad-\frac{8 \cdot \sqrt{11} \cdot(12)^{9 / 2}}{4 \cdot(12)^{3 / 2} \cdot(14)^{5 / 2} \cdot 19 \cdot 25} \cdot \frac{(16)^{4}}{(999)^{2}} \cdot\left(e_{5} / e_{4.5}\right)^{3} \cdot \frac{525768874}{(12)^{6}} \\
=\frac{2^{19 / 2} \cdot 3 \cdot 17 \cdot 1489}{5^{2} \cdot 7^{3 / 2} \cdot(11)^{5 / 2} \cdot(19)^{3 / 2}}-\frac{2^{19 / 2} \cdot(11)^{1 / 2} \cdot 13836023}{3^{7} \cdot 5^{2} \cdot 7^{5 / 2} \cdot(111)^{2}} \cdot\left(e_{5} / e_{4.5}\right)^{3}
\end{aligned}
$$

When $16 \leqq n<18\left(\frac{1}{18}<u \leqq \frac{1}{16}\right)$, we have

(the left hand side of $(10.6))<$

$$
\begin{aligned}
& \frac{21 \cdot 3017736 \cdot(16)^{7}}{3 \cdot(16)^{5} \cdot(18)^{3 / 2} \cdot(27)^{3 / 2} \cdot(15)^{5 / 2} \cdot(33)^{3 / 2}} \\
& -\frac{12 \cdot \sqrt{15} \cdot(16)^{9 / 2}}{4 \cdot(16)^{3 / 2} \cdot(18)^{5 / 2} \cdot 27 \cdot 33} \cdot \frac{(18)^{4}}{(1267)^{2}} \cdot\left(e_{17 / 3} / e_{31 / 6}\right)^{3} \cdot \frac{3275894178}{(16)^{6}} \\
& =\frac{2^{19 / 2} \cdot 7 \cdot 4657}{3^{15 / 2} \cdot 5^{5 / 2} \cdot(11)^{3 / 2}}-\frac{3^{15 / 2} \cdot 5^{1 / 2} \cdot 748947}{2^{19 / 2} \cdot 7^{2} \cdot 11 \cdot(181)^{2}} \cdot\left(e_{17 / 3} / e_{31 / 6}\right)^{3} .
\end{aligned}
$$

When $n \geqq 18\left(0<u \leqq \frac{1}{18}\right)$, we have

(the left hand side of $(10.6))<$

$$
\begin{aligned}
& \frac{23 \cdot 4843444 \cdot(18)^{7}}{3 \cdot(18)^{5} \cdot(20)^{3 / 2} \cdot(31)^{3 / 2} \cdot(17)^{5 / 2} \cdot(37)^{3 / 2}} \\
& \quad-\frac{14 \sqrt{17 \cdot} \cdot(18)^{9 / 2}}{4 \cdot(18)^{3 / 2} \cdot(20)^{5 / 2} \cdot 31 \cdot 37} \cdot \frac{1}{4^{2}} \cdot 1 \cdot \frac{6863844478}{(18)^{6}} \\
& =\frac{2 \cdot 3^{3} \cdot 23 \cdot 1210861}{5^{3 / 2} \cdot(17)^{5 / 2} \cdot(31)^{3 / 2} \cdot(37)^{3 / 2}}-\frac{7 \cdot(17)^{1 / 2} \cdot 3431922239}{2^{12} \cdot 3^{6} \cdot 5^{5 / 2} \cdot 31 \cdot 37}
\end{aligned}
$$

\section{$\S 15$. Proof of Theorem $C$ for $n \geqq 18$.}

In this section, we shall prove that Conjecture $\mathrm{C}$ is true for $n \geqq 18$. We suppose $n \geqq 18$ in this section. By means of (12.5), (13.3), (13.8) and (13.12), we obtain the following inequality

$$
\begin{gathered}
\left\{\frac{x^{2} \sqrt{n-x} F_{2}(x)}{(1-x)^{5}}+\frac{X^{2} \sqrt{n-X} F_{2}(X)}{(X-1)^{5}}\right\} \cdot\{\tilde{\lambda}(X)-\tilde{\lambda}(\beta)\}>B n^{2} \sqrt{n-1} \\
\quad \times\left[\frac{2^{11 / 2} \cdot 7^{2} \cdot(17)^{7 / 2} \cdot 307 \cdot(319)^{1 / 2}}{3^{3} \cdot(37)^{3 / 2} \cdot(46981)^{2}}+\frac{\sqrt{31 \cdot 38617 \cdot 761243}}{2^{15} \cdot 3^{6} \cdot 5^{2} \cdot(17)^{2} \cdot 37}\right]
\end{gathered}
$$




$$
\times \frac{1}{3^{2} \cdot 17} \cdot \frac{105379 \beta-123246}{2772-307 \beta} \quad \text { for } \quad \alpha_{1}(n) \leqq x<1 .
$$

Now, we do some numerical computation as follows.

(i ) $\frac{2^{11 / 2} \cdot 7^{2} \cdot(17)^{5 / 2} \cdot 307 \cdot(319)^{1 / 2}}{3^{5} \cdot(37)^{3 / 2} \cdot(46981)^{2}}$

$$
\begin{aligned}
& =\frac{2^{5} \cdot 7^{2} \cdot(17)^{2} \cdot 307}{3^{5} \cdot 37 \cdot(46981)^{2}} \times \sqrt{\frac{2 \cdot 17 \cdot 319}{37}}=\frac{32 \cdot 49 \cdot 289 \cdot 307}{243 \cdot 37 \cdot(46981)^{2}} \times \sqrt{\frac{2 \cdot 17 \cdot 319}{37}} \\
& >\frac{7.0101896}{10^{6}} \times 17.1211896>\frac{1.2002278}{10^{4}} .
\end{aligned}
$$

(ii) $\frac{\sqrt{31} \cdot 38617 \cdot 761243}{2^{15} \cdot 3^{8} \cdot 5^{2} \cdot(17)^{3} \cdot 37}=\frac{1}{10^{2}} \times \frac{38617 \cdot 761243}{8192 \cdot 6561 \cdot 4913 \cdot 37} \times \sqrt{31}$

$$
>\frac{1}{10^{2}} \times \frac{3.0088003}{10^{3}} \times 5.5677643>\frac{1.67522908}{10^{4}}
$$

(iii) $\quad \beta(n) \geqq \beta(18)=\frac{-1+\sqrt{613}}{17}=1.3975786 \cdots>1.3975786 \quad$ (by $\left.\quad(11.6)\right)$.

(iv) $\quad \frac{105379 \beta-123246}{2772-307 \beta}>\frac{147275.4352894-123246}{2772-429.0566302}$

$$
=\frac{24029.4352894}{2342.9433698}=10.25608881 \cdots>10.2560888 \text {. }
$$

By means of the above evaluations, we obtain

$$
\begin{aligned}
& \left\{\frac{x^{2} \sqrt{n-x} F_{2}(x)}{(1-x)^{5}}+\frac{X^{2} \sqrt{n-X} F_{2}(X)}{(X-1)^{5}}\right\} \cdot\{\tilde{\lambda}(X)-\tilde{\lambda}(\beta)\} \\
& >\frac{B n^{2} \sqrt{n-1}}{10^{4}}[1.2002278+1.67522908] \times 10.2560888 \\
& >\frac{B n^{2} \sqrt{n-1}}{10^{4}} \times 29.4909411 .
\end{aligned}
$$

Thus, we have proved the following

LEMMA 15.1. When $n \geqq 18$, we have the inequality

$$
\begin{aligned}
& \left\{\frac{x^{2} \sqrt{n-x} F_{2}(x)}{(1-x)^{5}}+\frac{X^{2} \sqrt{n-X} F_{2}(X)}{(X-1)^{5}}\right\}\{\tilde{\lambda}(X)-\tilde{\lambda}(\beta)\} \\
& >B n^{2} \sqrt{n-1} \times \frac{29.4909411}{10^{4}} \quad \text { for } \alpha_{1}(n) \leqq x<1,
\end{aligned}
$$

where $X=X(x)$.

Next we shall do analogous evaluations for the function $V(x, X(x))$, which 
is more fine than the ones done in $\S 10$. By means of (9.5), (14.3), (14.6) and (14.9), we obtain the following inequality

$$
\begin{array}{r}
\frac{V(x, X(x))}{s B \sqrt{n-1}}>\left[\frac{2^{19 / 2} \cdot 3^{4} \cdot 7^{3} \cdot(17)^{3 / 2} \cdot 307 \cdot(319)^{1 / 2}}{5 \cdot(23)^{2} \cdot 31 \cdot(46981)^{2}}+\frac{7 \cdot 38617 \cdot 761243}{2^{29 / 2} \cdot 3^{15} \cdot 5}\right] n \\
-\frac{s+t}{s}\left[\frac{2 \cdot 3^{3} \cdot 23 \cdot 1210861}{5^{3 / 2} \cdot(17)^{5 / 2} \cdot(31)^{3 / 2} \cdot(37)^{3 / 2}}-\frac{7 \cdot(17)^{1 / 2} \cdot 3431922239}{2^{12} \cdot 3^{6} \cdot 5^{5 / 2} \cdot 31 \cdot 37}\right] \\
\text { for } \alpha_{1}(n) \leqq x<1
\end{array}
$$

Now, we do some numerical computations as follows.

(ii)

$$
\begin{aligned}
& \frac{2^{19 / 2} \cdot 3^{4} \cdot 7^{3} \cdot(17)^{3 / 2} \cdot 307 \cdot(319)^{1 / 2}}{5 \cdot(23)^{2} \cdot 31 \cdot(46981)^{2}} \\
& \quad=\frac{1}{10} \times \frac{1024 \cdot 81 \cdot 343 \cdot 17 \cdot 307}{529 \cdot 31 \cdot(46981)^{2}} \times \sqrt{ } 2 \cdot 17 \cdot 319 \\
& \quad>\frac{1}{10} \times \frac{4.1020836}{10^{3}} \times 104.1441309>\frac{427.2079314}{10^{4}} . \\
& \frac{7 \cdot 38617 \cdot 761243}{2^{29 / 2} \cdot 3^{15} \cdot 5}=\frac{1}{10} \times \frac{7 \cdot 38617 \cdot 761243}{16384 \cdot 14348907} \times \sqrt{2} \\
& >\frac{1}{10} \times \frac{8.75308456}{10} \times 1.41421356>\frac{1237.8730876}{10^{4}} .
\end{aligned}
$$

(iii)

$$
2 \cdot 3^{3} \cdot 23 \cdot 1210861
$$

$$
\begin{aligned}
& 5^{3 / 2} \cdot(17)^{5 / 2} \cdot(31)^{3 / 2} \cdot(37)^{3 / 2} \\
& =\frac{1}{10^{2}} \times \frac{8 \cdot 27 \cdot 23 \cdot 1210861}{4913 \cdot 961 \cdot 1369} \times \sqrt{ } 5 \cdot 17 \cdot 31 \cdot 37 \\
& <\frac{1}{10^{2}} \times \frac{9.3068407}{10} \times 312.2418935<2.905985563 .
\end{aligned}
$$

(iv)

$$
\begin{gathered}
\frac{7 \cdot(17)^{1 / 2} \cdot 3431922239}{2^{12} \cdot 3^{6} \cdot 5^{5 / 2} \cdot 31 \cdot 37}=\frac{1}{10^{3}} \times \frac{7 \cdot 3431922239}{512 \cdot 729 \cdot 31 \cdot 37} \times \sqrt{ } 5 \cdot 17 \\
>\frac{1}{10^{3}} \times 56.11443206 \times 9.21954445>0.517349500 .
\end{gathered}
$$

Making use of the above evaluations, we obtain

$$
\begin{gathered}
\frac{V(x, X(x))}{s B \sqrt{n-1}}>\frac{1}{10^{4}} \times[427.2079314+1237.8730876] n \\
\quad-\frac{s+t}{s}[2.905985563-0.517349500] \\
=\frac{1665.081019}{10^{4}} n-\frac{23886.36063}{10^{4}} \frac{s+t}{s} .
\end{gathered}
$$


Thus, we have proved the following

LEMMA 15.2. When $n \geqq 18$, we have the inequality

$$
\begin{array}{r}
V(x, X(x))>\frac{s B \sqrt{n-1}}{10^{4}} \times\left(1665.081019 n-23886.36063 \frac{s+t}{s}\right) \\
\text { for } \alpha_{1}(n) \leqq x<1 .
\end{array}
$$

Proof of Theorem $C$ for $n \geqq 18$. By means of (11.8), Lemma 15.1 and Lemma 15.2, we have the inequality

$$
\begin{aligned}
& V(x, \beta)>\frac{s B \sqrt{n-1}}{10^{4}} \\
& \times\left[29.4909411 \frac{n^{2}}{s}+1665.081019 n-23886.36063 \frac{s+t}{s}\right] \\
& \text { for } \alpha_{1}(n) \leqq x<1 .
\end{aligned}
$$

Since we have the following inequality for $\alpha_{1}(n) \leqq x<1$

$$
\frac{1}{s}=\frac{1}{X(x)-1}>-\frac{1}{(2 n+10) /(2 n+1)-1}=\frac{2 n+1}{9}, \frac{t}{s}=\frac{1-x}{X(x)-1}<1
$$

sy means of Lemma 6.2 and (6.1), we obtain

$$
\begin{aligned}
29 . & 4909411 \frac{n^{2}}{s}+1665.081019 n-23886.36063 \frac{s+t}{s} \\
& >\frac{29.4909411}{9} n^{2}(2 n+1)+1665.081019 n-47772.72126 \\
> & 3.27677 n^{2}(2 n+1)+1665.08101 n-47772.72126 \\
& \geqq 3.27677 \times(18)^{2} \times 37+1665.08101 \times 18-47772.72126 \\
& =39281.91876+29971.45818-47772.72126>0 .
\end{aligned}
$$

Thus, when $n \geqq 18$, we obtain the inequality (11.7)

$$
V(x, \beta)>0 \quad \text { for } \quad \alpha_{1}(n) \leqq x<1,
$$

vhich implies Theorem $\mathrm{C}$ for $n \geqq 18$ by the reason stated in $\S 11$.

\section{$\S 16$. Proof of Theorem $C$ for $16 \leqq n<18$.}

In this section, we shall prove that Conjecture $\mathrm{C}$ is true for $16 \leqq n<18$ by he same way as in $\S 15$. We suppose $16 \leqq n<18$ through this section. By neans of (12.5), (13.2), (13.7) and (13.11), we obtain the following inequality

$$
\left\{\frac{x^{2} \sqrt{n-x} F_{2}(x)}{(1-x)^{5}}+-\frac{X^{2} \sqrt{n-X} F_{2}(X)}{(X-1)^{5}}\right\}\{\tilde{\lambda}(X)-\tilde{\lambda}(\beta)\}>B n^{2} \sqrt{n-1}
$$




$$
\begin{aligned}
& \times\left[\frac{2^{11 / 2} \cdot 3^{2} \cdot 5^{7 / 2} \cdot 7^{1 / 2} \cdot 241}{(11)^{3 / 2} \cdot(11019)^{2}}+\frac{2933 \cdot 10409}{2^{41 / 2} \cdot 3^{5 / 2} \cdot 5 \cdot 11} \cdot \frac{e_{17} \cdot\left(e_{9}\right)^{2}}{\sqrt{e_{36} \cdot\left(e_{6.2}\right)^{5 / 2}}}\right] \\
& \times \frac{2^{2}}{3^{3} \cdot 5} \cdot \frac{16353 \beta-19728}{1936-241 \beta} \text { for } \alpha_{1}(n) \leqq x<1 .
\end{aligned}
$$

Now, we do some numerical computations as follows.

(i ) $\frac{2^{15 / 2} \cdot 5^{5 / 2} \cdot 7^{1 / 2} \cdot 241}{3 \cdot(11)^{3 / 2} \cdot(11019)^{2}}=\frac{10^{2} \cdot 32 \cdot 241}{3 \cdot 121 \cdot(11019)^{2}} \times \sqrt{2 \cdot 5 \cdot 7 \cdot 11}$

$$
>10^{2} \times \frac{1.7497501}{10^{7}} \times 27.7488738>\frac{4.8553594}{10^{4}} .
$$

(ii) $\frac{2933 \cdot 10409}{2^{37 / 2} \cdot 3^{11 / 2} \cdot 5^{2} \cdot 11}=\frac{1}{10^{2}} \times \frac{2933 \cdot 10409}{131072 \cdot 729 \cdot 11} \times \sqrt{ } 6$

$$
>\frac{1}{10^{2}} \times-\frac{2.9046307}{10^{2}} \times 2.4494897>\frac{7.1148629}{10^{4}} .
$$

(iii) $\frac{e_{17} \cdot\left(e_{9}\right)^{2}}{\sqrt{e_{36} \cdot\left(e_{6.2}\right)^{5 / 2}}}=\frac{(18 / 17)^{17} \cdot(10 / 9)^{18}}{(37 / 36)^{18} \cdot(72 / 62)^{15.5}}>\frac{2.6424143 \times 6.6624633}{1.6375178 \times 10.1527224}$

$$
>\frac{17.6049882}{16.6252637}=1.05892986 \cdots>1.0589298 \text {. }
$$

(iv) $\frac{7.1148629}{10^{4}} \times 1.0589298>\frac{7.5341403}{10^{4}}$.

(v) $\beta(n) \geqq \beta(16)=\frac{-1+\sqrt{ } 481}{15}=1.3954474 \cdots>1.3954474 \quad$ (by $\left.(11.6)\right)$.

(vi) $\frac{16353 \beta-19728}{1936-241 \beta}>\frac{22819.7513322-19728}{1936-336.3028234}=\frac{3091.7513322}{1599.6971766}$

$$
=1.932710376 \cdots>1.93271037 \text {. }
$$

By means of the above evaluations, we obtain

$$
\begin{aligned}
& \left\{\frac{x^{2} \sqrt{n-x} F_{2}(x)}{(1-x)^{5}}+\frac{X^{2} \sqrt{n-X} F_{2}(X)}{(X-1)^{5}}\right\}\{\tilde{\lambda}(X)-\tilde{\lambda}(\beta)\} \\
& >\frac{B n^{2} \sqrt{n-1}}{10^{4}} \times[4.8553594+7.5341403] \times 1.93271037 \\
& >\frac{B n^{2} \sqrt{n-1}}{10^{4}} \times 23.9453145 .
\end{aligned}
$$

Thus, we have proved the following

LEMMA 16.1. When $16 \leqq n<18$, we have the inequality 


$$
\begin{gathered}
\left\{\frac{x^{2} \sqrt{n-x} F_{2}(x)}{(1-x)^{5}}+\frac{X^{2} \sqrt{n-X} F_{2}(X)}{(X-1)^{5}}\right\}\{\tilde{\lambda}(X)-\tilde{\lambda}(\beta)\} \\
>B n^{2} \sqrt{n-1} \times \frac{23.9453145}{10^{4}} \quad \text { for } \quad \alpha_{1}(n) \leqq x<1,
\end{gathered}
$$

where $X=X(x)$.

Next, by means of (9.5), (14.2), (14.5) and (14.8) we obtain the following inequality

$$
\begin{aligned}
& \frac{V(x, X(x))}{s B \sqrt{n-1}>\left[\frac{2^{3} \cdot 3^{7 / 2} \cdot 5^{5 / 2} \cdot 7^{3 / 2} \cdot(37)^{3 / 2} \cdot 241}{(319)^{2} \cdot(11019)^{2}}+\frac{2^{20} \cdot 5^{3 / 2} \cdot(37)^{3 / 2} \cdot 181 \cdot 241}{3^{29 / 2} \cdot 7^{1 / 2} \cdot(11019)^{2}}\right.} \\
& \left.\quad+\frac{2933 \cdot 10409}{2^{23 / 2} \cdot 3^{9 / 2} \cdot 5^{4}} \cdot \frac{e_{17} \cdot\left(e_{9}\right)^{2}}{\sqrt{e_{36}} \cdot\left(e_{6.2}\right)^{5 / 2}}\right] n \\
& \quad-\frac{s+t}{s}\left[-\frac{2^{19 / 2} \cdot 7 \cdot 4657}{3^{15 / 2} \cdot 5^{5 / 2} \cdot(11)^{3 / 2}}-\frac{3^{15 / 2} \cdot 5^{1 / 2} \cdot 748947}{2^{19 / 2} \cdot 7^{2} \cdot 11 \cdot(181)^{2}} \cdot\left(\frac{e_{17 / 3}}{e_{31 / 6}}\right)^{3}\right] \\
& \quad \text { for } \alpha_{1}(n) \leqq x<1 .
\end{aligned}
$$

Now, we do some numerical computations as follows.

(i ) $\frac{2^{3} \cdot 3^{7 / 2} \cdot 5^{5 / 2} \cdot 7^{3 / 2} \cdot(37)^{3 / 2} \cdot 241}{(319)^{2} \cdot(11019)^{2}}=10^{2} \times \frac{2 \cdot 27 \cdot 7 \cdot 37 \cdot 241}{(319)^{2} \cdot(11019)^{2}} \times \sqrt{ } 3 \cdot 5 \cdot 7 \cdot 37$

$$
>\frac{2.7280029}{10^{5}} \times 62.3297681>\frac{17.0035788}{10^{4}} \text {. }
$$

(ii) $\frac{2^{20} \cdot 5^{3 / 2} \cdot(37)^{3 / 2} \cdot 181 \cdot 241}{3^{29 / 2} \cdot 7^{1 / 2} \cdot(11019)^{2}}=10 \times \frac{524288 \cdot 37 \cdot 181 \cdot 241}{14348907 \cdot 7 \cdot(11019)^{2}} \times \sqrt{ } 3 \cdot 5 \cdot 7 \cdot 37$

$$
>\frac{6.9385070}{10^{4}} \times 62.3297681>-\frac{432.4755322}{10^{4}} .
$$

(iii) $\frac{2933 \cdot 10409}{2^{23 / 2} \cdot 3^{9 / 2} \cdot 5^{4}}=\frac{1}{10^{4}} \times \frac{2933 \cdot 10409}{256 \cdot 243} \times \sqrt{ } 2 \cdot 3$

$$
>\frac{1}{10^{4}} \times 490.7664127 \times 2.4494897>\frac{12.0212727}{10^{2}}
$$

and

$$
\frac{2933 \cdot 10409}{2^{23 / 2} \cdot 3^{9 / 2} \cdot 5^{4}} \times \frac{e_{17} \cdot\left(e_{9}\right)^{2}}{\sqrt{e_{36}} \cdot\left(e_{6.2}\right)^{5 / 2}}>\frac{12.0212727}{10^{2}} \times 1.0589298>\frac{1272.9683895}{10^{4}} .
$$

(iv) $\frac{2^{19 / 2} \cdot 7 \cdot 4657}{3^{15 / 2} \cdot 5^{5 / 2} \cdot(11)^{3 / 2}}=\frac{1}{10^{3}} \times \frac{4096 \cdot 7 \cdot 4657}{6561 \cdot 121} \times \sqrt{2 \cdot 3 \cdot 5 \cdot 11}$

$$
<\frac{168.1933489}{10^{3}} \times 18.1659022<3.055383927 .
$$


( v ) $\frac{3^{15 / 2} \cdot 5^{1 / 2} \cdot 748947}{2^{19 / 2} \cdot 7^{2} \cdot 11 \cdot(181)^{2}}=-\frac{2187 \cdot 748947}{1024 \cdot 49 \cdot 11 \cdot(181)^{2}} \times \sqrt{ } 2 \cdot 3 \cdot 5$ $>\frac{9.05845219}{10^{2}} \times 5.47722557>0.496151859$.

(vi) $\left(\frac{e_{17 / 3}}{e_{31 / 6}}\right)^{3}=\left(\frac{20}{17}\right)^{17} /\left(\frac{37}{31}\right)^{31 / 2}>\frac{15.8444899}{15.5246018}=1.020605237 \cdots$

$$
>1.020605237 \text {. }
$$

(vii) $\frac{3^{15 / 2} \cdot 5^{1 / 2} \cdot 748947}{2^{19 / 2} \cdot 7^{2} \cdot 11 \cdot(181)^{2}} \times\left(\frac{e_{17 / 3}}{e_{31 / 6}}\right)^{3}>0.496151895 \times 1.020605237>0.506375185$.

Making use of the above evaluations, we obtain

$$
\begin{aligned}
& \frac{V(x, X(x))}{s B \sqrt{n-1}}>-\frac{1}{10^{4}} \times[17.0035788+432.4755322+1272.9683895] n \\
& \quad-\frac{s+t}{s}[3.055383927-0.506375185]=\frac{1722.4475005}{10^{4}} n-\frac{25490.08742}{10^{4}}-\frac{s+t}{s} .
\end{aligned}
$$

Thus, we have proved the following

LEMMA 16.2. When $16 \leqq n<18$, we have the inequality

$$
\begin{array}{r}
V(x, X(x))>\frac{s B \sqrt{n-1}}{10^{4}} \times\left(1722.4475 n-25490.08742 \frac{s+t}{s}\right) \\
\text { for } \alpha_{1}(n) \leqq x<1 .
\end{array}
$$

Proof of Theorem $C$ for $16 \leqq n<18$. By means of (11.8), Lemma 16.1 and Lemma 16.2, we have the inequality (16.3) $V(x, \beta)$

$$
\begin{array}{r}
>\frac{s B \sqrt{n-1}}{10^{4}} \times\left[23.9453145 \frac{n^{2}}{s}+1722.4475 n-25490.08742 \frac{s+t}{s}\right] \\
\text { for } \alpha_{1}(n) \leqq x<1 .
\end{array}
$$

By the same reason as in $\S 15$, we have

$$
\begin{aligned}
& 23.9453145 \frac{n^{2}}{s}+1722.4475 n-25490.08742 \frac{s+t}{s} \\
& >\frac{23.9453145}{9} n^{2}(2 n+1)+1722.4475 n-25490.08742 \times 2 \\
& >2.66059 n^{2}(2 n+1)+1722.4475 n-50980.17484 .
\end{aligned}
$$

Now, for the last cubic polynome of $n$ we have

$$
\left[2.66059 n^{2}(2 n+1)+1722.4475 n-50980.17484\right]_{n=16}
$$


and $=22476.66432+27559.16-50980.17484=-944.35052<0$

$\left[2.66059 n^{2}(2 n+1)+1722.4475 n-50980.17484\right]_{n=16.1}$ $\doteq 22896.43093+27731.40475-50980.17484=-352.33916<0$

and then

$\left[2.66059 n^{2}(2 n+1)+1722.4475 n-50980.17484\right]_{n=16.2}$ $>23321.39100+27903.6495-50980.17484=244.86566>0$.

Hence we see that when $n \geqq 16.2$ we obtain the inequality (11.7)

$$
V(x, \beta)>0 \quad \text { for } \quad \alpha_{1}(n) \leqq x<1,
$$

which implies Theorem $\mathrm{C}$ for $16.2 \leqq n<18$.

Now, in order to prove (11.7) for $16 \leqq n<16.2$ we put

$$
\alpha_{2}(n)=X_{n}^{-1}\left(\frac{2 n+9}{2 n+1}\right) \text {. }
$$

For $\alpha_{2}(n) \leqq x<1$, we have

$$
\frac{1}{s} \geqq \frac{2 n+1}{8}, \quad \frac{s+t}{s}<2
$$

and so

$23.9453145 \frac{n^{2}}{s}+1722.4475 n-25490.08742 \frac{s+t}{s}$

$>\frac{23.9453145}{8} n^{2}(2 n+1)+1722.4475 n-25490.08742 \times 2$

$>2.99316 n^{2}(2 n+1)+1722.4475 n-50980.17484$

$\geqq 2.99316 \times(16)^{2} \times 33+1722.4475 \times 16-50980.17484$

$=25286.21586+27559.16-50980.17484=1865.20084>0$.

Then, for $\alpha_{1}(n) \leqq x<\alpha_{2}(n)$, we evaluate $\frac{s+t}{s}$. By Proposition 4 in (IV), $\S 9$, $\frac{X(x)-1}{1-x}$ is decreasing with respect to $x$ for $0<x<1$, hence we have

$$
1<\frac{s+t}{s}<\left[\frac{s+t}{s}\right]_{x=\alpha_{2}(n)} \quad \text { for } \quad \alpha_{1}(n) \leqq x<\alpha_{2}(n)
$$

and

$$
\left[\frac{s+t}{s}\right]_{x=\alpha_{2}(n)}=1+\frac{1-\alpha_{2}(n)}{8 /(2 n+1)}=1+\frac{2 n+1}{8}\left(1-\alpha_{2}(n)\right),
$$

i.e. 


$$
-\frac{s+t}{s}<\frac{2 n+9}{8}-\frac{2 n+1}{8} \alpha_{2}(n) .
$$

By means of Proposition 1 in (IV), §2, we have

$$
n-(n-1)\left(\alpha_{2}(n)\right)^{1 /(n-1)}<\frac{2 n+9}{2 n+1},
$$

hence

$$
\left(\frac{2 n^{2}-n-9}{2 n^{2}-n-1}\right)^{n-1}<\alpha_{2}(n)
$$

From (16.4) and (16.5), we obtain

$$
\frac{s+t}{s}<\frac{2 n+9}{8}-\frac{2 n+1}{8}\left(\frac{2 n^{2}-n-9}{2 n^{2}-n-1}\right)^{n-1} .
$$

$\frac{2 n^{2}-n-9}{2 n^{2}-n-1}$ is monotone increasing with respect to $n$, and hence from (16.6) we obtain for $16 \leqq n<16.2$

$$
\begin{aligned}
\frac{s+t}{s} & <\frac{2 \times 16.2+9}{8}-\frac{2 \times 16+1}{8} \cdot\left(\frac{2 \times(16)^{2}-16-9}{2 \times(16)^{2}-16-1}\right)^{16.2-1} \\
& =\frac{41.4}{8}-\frac{33}{8} \cdot\left(\frac{487}{495}\right)^{15.2}<5.175-4.125 \times 0.7806227 \\
& =5.175-3.2200686375=1.9549313625<1.96 .
\end{aligned}
$$

Making use of this evaluation, for $\alpha_{1}(n) \leqq x<\alpha_{2}(n)$ we have

$$
\begin{aligned}
& 23.9453145 \frac{n^{2}}{s}+1722.4475 n-25490.08742 \frac{s+t}{s} \\
&> \frac{23.9453145}{9} n^{2}(2 n+1)+1722.4475 n-25490.08742 \times 1.96 \\
&> 2.66059 n^{2}(2 n+1)+1722.4475 n-49960.5713432 \\
& \geqq 2.66059 \times(16)^{2} \times 33+1722.4475 \times 16-49960.5713432 \\
&=22476.66432+27559.16-49960.5713432=75.2529768>0 .
\end{aligned}
$$

Thus, we have proved that when $16 \leqq n<16.2$ the inequality (11.7) is also true for $\alpha_{1}(n) \leqq x<1$. This fact implies that Conjecture $\mathrm{C}$ is ture for $16 \leqq n<16.2$.

From the observations in $\S 15$ and $\S 16$, we obtain the main theorem.

THEOREM. C. The period function $T$ as a function of $\tau$ and $n$ is monotone decreasing with respect to $n \geqq 16$ for any fixed $\tau(0<\tau<1)$.

Remark. In fact, Conjecture $\mathrm{C}$ is natural for small values of $n(2<n<30)$ 
from the numerical data obtained by M. Urabe (see Fig. 9 in [6]). From the arguments in $\S 16$, it seems to the author that in order to prove Theorem $\mathrm{C}$ for $2<n<16$ we have to develope another and more fine way than the ones in this paper.

\section{REFERENCES}

[1] S.S. Chern, M. do Carmo and S. Kobayashi, Minimal submanifolds of a sphere with second fundamental form of constant length, Functional Analysis and Related Fields, Springer-Verlag, (1970), 60-75.

[2] S. FuRUYA, On periods of periodic solutions of a certain nonlinear differential equation, Japan-United States Seminar on Ordinnary Differential and Functional Equations, Lecture Notes in Mathematics, Springer-Verlag, 243 (1971), 320-323.

[3] W. Y. Hsiang and H.B. Lawson, JR., Minimal submanifolds of low cohomogeneity, J. Diff. Geometry, 5 (1970), 1-38.

[4] T. OTsUKI, Minimal hypersurfaces in a Riemannian manifold of constant curvature, Amer. J. Math. 92 (1970), 145-173.

[5] T. Oтsuki, On integral inequalities related with a certain nonlinear differential equation, Proc. Japan Acad., 48 (1972), 9-12.

[6] T. Otsuki, On a 2-dimensional Riemannian manifold, Differential Geometry, in honor of K. Yano, Kinokuniya, Tokyo, (1972), 401-414.

[7] T. Otsuki, On a family of Riemannian manifolds defined on an $m$-disk, Math. J. Okayama Univ., 16 (1973), 85-97.

[8] T. OTsuki, On a bound for periods of solutions of a certain nonlinear differential equation (I), J. Math. Soc. Japan, 26 (1974), 206-233.

[9] T. Otsuki, On a bound for periods of solutions of a certain nonlinear differential equation (II), Funkcialaj Ekvacioj, 17 (1974), 193-205.

[10] T. Oтsuki, Geodesics of $O_{n}^{2}$ and an analysis on a related Riemann surface, Tôhoku Math. J., 28 (1976), 411-427.

[11] T. OTsuki, A certain property of geodesics of the family of Riemannian manifolds $O_{n}^{2}$ (I), Proc. of Japan-United States Seminar on Minımal Submanifolds, including Geodesics, Kaigai, Tokyo, (1978), 173-192.

[12] T. Otsuki, A certain property of geodesics of the family of Riemannian man1folds $O_{n}^{2}$ (II), Kodai Math. J., 2 (1979), 211-242.

[13] T. Otsuki, A certain property of geodesics of the family of Riemannian manifolds $O_{n}^{2}$ (III), Kodai Math. J., 4 (1981), 28-70.

[14] T. OTsuki, A certain property of geodesics of the family of Riemannian manifolds $O_{n}^{2}$ (IV), Kodai Math. J., 5 (1982), 160-199.

[15] M. MAeda AND T. Otsuki, Models of the Riemannian manifolds $O_{n}^{2}$ in the Lorentzian 4-spaces, J. Diff. Geometry, 9 (1974), 97-108.

[16] M. URABE, Computations of periods of a certain nonlinear autonomous osc1lations, Study of algorithms of numerical computations, Sûrikaiseki Kenkyûsho Kôkyû-roku, 149 (1972), 111-129 (Japanese).

Department of Mathematics

SCIENCE University OF TOKYO

WakamiYa-cho 26 , ShinjukU-Ku

TOKYO, JAPAN 162 